\title{
Investigation of therapeutic effect of Longchai Formula Granule on chronic hepatitis B patients: A double-blind, controlled and randomized clinical trial
}

\author{
Zhanpeng Yan ${ }^{1,2}$, Tingting $\mathrm{Xu}^{1,2}$, Kelei Su${ }^{1,2}$, Chi Wang ${ }^{3}$, Tong Yan ${ }^{4}$, Zhong Hua ${ }^{5}$, Erli Gu ${ }^{6}$ and \\ Fangshi Zhu ${ }^{1,2, a}$ \\ ${ }^{1}$ Affiliated Hospital of Traditional Chinese and Western Medicine, Nanjing University of Chinese Medicine, Nanjing \\ 210028, China \\ 2Jiangsu Province Institute of Traditional Chinese Medicine, Nanjing 210028, China \\ ${ }^{3}$ Taizhou Integrated Hospital of Traditional Chinese and Western Medicine, Taizhou 225300, China \\ ${ }^{4}$ Changzhou Third People's Hospital, Changzhou 213001, China \\ ${ }^{5}$ Wuxi Infectious Diseases Hospital, Wuxi 214005, China \\ ${ }^{6}$ Nantong Third People's Hospital, Nantong 226000, China
}

\begin{abstract}
Objective: To objectively evaluate the clinical efficacy of Longchai Formula Granule (LCFG) on chronic hepatitis B (CHB). Methods: According to GCP, $100 \mathrm{CHB}$ patients who meet enter criterion were encoded and divided randomly into the test group (50 cases) and the control group (50 cases). The patients in test group were treated with LCFG and matrine capsule simulation agent, the patients in control group was treated with matrine capsule and LCFG simulation agent. Both groups have 12-week treatment period. The changes of Chinese medicine symptoms, ALT, AST, graduation of liver function, $\mathrm{HBeAg}, \mathrm{HBsAg}, \mathrm{HBV}-$ DNA in CHB patients were observed and compared. Results: After unblinding, statistical data showed that there were 82 effective actual CHB cases composed of $40 \mathrm{CHB}$ patients in test group (10 cases dropout) and $42 \mathrm{CHB}$ patients in control group ( 8 cases dropout). The total efficient of Chinese symptoms improvement and ALT in test group is better than the control group $(P<0.05)$. The contents of AST, the graduation of liver function, HBeAg, HBsAg, and HBV-DNA in two groups were totally improved than before treatment, but there was no significant differences between groups $(P>0.05)$. As a whole, it shows a tendency that the change of these values in test group induced by LCFG is better than these values in control group induced by matrine capsule. Conclusions: In summary, LCFG is effective for CHB patients and has no significant difference with matrine cpasules. However, LCFG is better than matrine capsule in the improvement of symptomatology and liver function.
\end{abstract}

\section{Introduction}

Hepatitis B virus (HBV) infection witch infected is a serious global health problem, and about 2 billion people were infected worldwide. Each year more than 300 thousand HBV carriers die of acute

\footnotetext{
${ }^{a}$ Correspondence to: Fangshi Zhu, E-mail: zhufangshi@126.com, Tel: +86-25-52632761, Address:

No.100, Shizi Street, Hongshan Road, Nanjing City, Jiangsu Province, China.

This work was supported by Scientific and Technological Innovation and Achievements

Transformation Special Projects of Jiangsu Province (BM2009903)

Trial registration: This study is registered at ClinicalTrials.gov, ChiCTR-IOR-14005585.
} 
hepatitis, liver fibrosis, liver cirrhosis and hepatocellular carcinoma (HCC) induced by chronic hepatitis B (CHB) [1-4]. Antiviral and liver-protecting therapy is a standardized treatment method that can reduce morbidity and mortality HBV infected patients[5-6]. Since a significant proportion of CHB patients fail to get satisfactory treatment from conventional medicine, so many CHB patients seek help from complementary and alternative medicine (CAM), especially traditional Chinese medicine (TCM)[7]. The Chinese pharmacopoeias contain numerous specific medicines and formulas, and numerous studies show that several herbs have antiviral activity and can prevent viral infection. Recently some clinical reports have suggested that the appropriate usage of TCM in combination with modern western medicine can prevent HBV infection and ameliorate symptoms of CHB patients, TCM have shown great potential prospects for treating CHB[8-10].

Longchai Formula (LCF) is an experienced and effective Chinese medicine formula developed by Professor JIN Shi, which is PhD tutor of Nanjing University of Chinese Medicine and the instructor inheriting the academic experience of Chinese famous physicians. LCF has affirmative treatment efficacy on CHB patients in clinical applications for many years, and LCF is good at improving symptoms, recovering liver function, decreasing HBV surface markers in CHB[11-12]. In our previous study, we have elaborated and discussed the theoretical mechanism of LCF on treating CHB[13]. LCF and LCF-containing serum could inhibit the growth of HepG2.2.15 cells and reduced the secretion level of HBsAg and HBeAg in vitro[14-15].Recent experiments show that LCF could increase drug sensitivity of nucleoside analogues (NA) treat CHB[16]. In order to objectively further evaluate the clinical efficacy of LCF on the treatment for CHB, a randomized, double-blinded, double-dummy and parallel-controlled trial was designed and implemented. The results are as follows.

\section{Methods}

\subsection{Patients}

One hundred CHB patients were selected from 5 hospitals, including Affiliated Hospital of Traditional Chinese and Western Medicine (Nanjing University of Chinese Medicine), Taizhou Integrated Hospital of Traditional Chinese and Western Medicine, Changzhou Third People's Hospital, Wuxi Infectious Diseases Hospital and Nantong Third People's Hospital, date from 30, December 2010 to 31, December 2012. 100 patients were divvied randomly into the test group and test group by statistics software SAS9.1. After unblinding of this clinical trail, there were 18 cases of dropouts and withdrawals, containing 10 cases in test group and 8 cases in control group (the expulsion rate $<20 \%$ ). The trial group was comprised 17 women and 23 men, age range 20-63 years (mean age is $38.45 \pm 14.28$ years), course of disease range $14-78$ months (mean course is $39.55 \pm 16.57$ months). The control group was comprised 16 women and 26 men, age range $25-61$ years (mean age is $38.45 \pm 14.28$ years), course of disease range 12-70 months (mean course is $34.78 \pm 15.79$ months). The $\chi^{2}$ and t test showed no significant difference in age, sex and course of disease. $(P>0.05)$.

\subsection{Inclusion criteria}

The inclusion criteria were as follows: (a) patients conforming to the diagnosis standard of chronic hepatitis B that defined in the Guidance of Prevention and Treatment of Chronic Hepatitis B updated in 2010;[17] (b) patients conforming to the TCM standard of "zheng" differentiation for chronic hepatitis B according to Guiding principle of clinical research on new drugs of Chinese medicine;[18] (c) patients with the age of 18-65 years and gen; (d) patients do not taking related medicine of treating CHB within half of month.

\subsection{Exclusion criteria}

The exclusion criteria were as follows: (a) patients aged below 18 or above 65 years old; (b) patients 
infected other type hepatitis virus at the same time; (c) patients had autoimmune hepatitis or hepatitis caused by drug intoxication and ethylism; (d) patients had been diagnosed with severe hepatitis, hepatic failure, cirrhosis, hepatocellular carcinoma or serious primary disease in cardiovascular, cerebrovascular, kidney, lung, endocrine system, hematopoietic system and mental disease; (e) patients were pregnant or lactating women; (f). patients were allergic physique or allergic to multiple drugs.

\subsection{Dropouts and withdrawals}

Participant in this clinical trail who did not complete the treatment programs had been excluded during the observation period.

\subsection{Treatment}

The CHB patients in test group were treated with LCFG (consisting of six medicinal herbs: Solanum nigrum L., Bupleurum chinense DC., Hedyotis diffusa Willd, Sedum sarmentosum Bunge, Gardenia jasminoides Ellis and Scutellaria baicalensis Georgi, and produced by Jiangyin Tianjiang Pharmaceutical Co., Ltd.), 2 times a day, 1/2 dose a time, infused with water. Meanwhile patients in test group were administrated with mimetic matrine capsules used for placebo-controlled (Jiangsu Province Engineering Research Center), 3 times a day, $0.2 \mathrm{~g}$ a time. The CHB patients in control group were treated with matrine capsules (containing 5\%medicine, starch, food coloring, bittering agent, and produced by Chia Tai Tianqing Pharmaceutical Co., Ltd.), 3 times a day, $0.2 \mathrm{~g}$ a time. Meanwhile patients in control group were administrated mimetic LCFG used for placebo-controlled (Jiangyin Tianjiang Pharmaceutical Co., Ltd.), 2 times a day, 1/2 dose a time, infused with water. Both groups have therapeutic course for 12 weeks, according to the rule that minimum observation time is no less than 3 months from the Guiding principle of clinical research on new drugs of Chinese medicine[18].

\subsection{Evaluation of efficacy}

At the time point of 0,6 and 12 weeks, the efficacy of drugs on CHB patients was evaluated comprehensively. Blood routine examination, urine routine examination, defecate routine examination, kidney function examination, electrocardiogram and chest examination were detected in order to drug safety. The contents of HBV-DNA, HBsAg, HbeAg, ALT and AST in serum of CHB patients were detected.

Symptom score was evaluated and composed of fatigue, liver pain, jaundice, hepatosplenomegaly, tongue, pulse and so on. According to the degree of symptoms CHB patients were divided into none, mild, moderate, and severe patients, and the symptom score were respectively recorded as $0,1,2$ and 3. In the end of trail the total scores of each CHB patient was calculated.

The assess standard of the damage degrees of liver function as follows: (a) mild damage, there are no or mild clinical symptoms, and only 1 or 2 kind liver function index appears mildly abnormal; (b) moderate damage, symptoms, signs and index from observe and laboratory tests is between mild damage and severe damage; (c) severe damage, there are significant or persistent hepatitis symptoms, such as fatigue, anorexia, abdominal distension, loose stools, hepatic face, liver palms, spider, splenomegaly, etc. The contents of serum ALT and/or AST in CHB patients were repeatedly or persistently elevated, content of serum albumin is decreased or the ratio of albumin/globulin $(A / G)$ is reversed, content of gamma globulin is significantly increased. In addition to the above abnormal changes, albumin is less than $32 \mathrm{~g} / \mathrm{L}$, total bilirubin is 5 times higher than the normal, prothrombin activity is more than $40 \%$ and less than $60 \%$, cholinesterase is less than $2500 \mathrm{U} / \mathrm{L}$, any one in the above four indexes happened could be diagnosed as chronic severe hepatitis. These principles were referenced to the Guiding principle of clinical research on new drugs of Chinese medicine. ${ }^{18}$

The assessment standard of efficacy on CHB as follows: (a) marked effect, the main symptoms 
negative; (b) normal effect, the main symptoms alleviated or disappeared, liver function is normal or has $50 \%$ reduction than before, one index among HBV-DNA, HBeAg and HBsAg was negative; (c) no effect, related symptoms and indexes have not ameliorated or even worsen. These principles were referenced to the Guiding principle of clinical research on new drugs of Chinese medicine[18].

The assessment standard of syndrome curative effect of TCM as follows: (a) clinical fully recovered, the main symptoms and signs disappeared or almost disappeared, and reduction of symptom score is above or equal $95 \%$; (b) marked effect, the main symptoms and signs improved significantly, and reduction of symptom score is between $70 \%$ and $95 \%$; (c) normal effect, the main symptoms and signs improved mildly, and reduction of symptom score is between $30 \%$ and $70 \%$; (d) no effect, main symptoms and signs no improved or worsen, reduction of symptom score is below $30 \%$. These principles were referenced to the Guiding principle of clinical research on new drugs of Chinese medicine[18].

\subsection{Statistical analysis}

Statistical software SAS 9.1 (SAS Institute Inc., USA) was used for data processing. Quantitative data are expressed as mean \pm standard deviation. For comparison of measured data between the two groups, the $t$ test of independent samples was used for date of homogeneity of variance. The Wilcoxon test was used for classified data. Statistical significance was assumed at $P<0.05$.

\section{Results}

\subsection{Comparison of TCM symptom improvement between two groups}

Table 1. Comparison of TCM symptom improvement between two groups.

\begin{tabular}{llccccc}
\hline Group & $\mathrm{n}$ & $\begin{array}{l}\text { Clinical } \\
\text { recovery }\end{array}$ & $\begin{array}{l}\text { Marked } \\
\text { effect }\end{array}$ & $\begin{array}{c}\text { Normal } \\
\text { effect }\end{array}$ & $\begin{array}{c}\text { No } \\
\text { effect }\end{array}$ & $\begin{array}{c}\text { Total } \\
\text { effective rate }\end{array}$ \\
\hline Test & 40 & 11 & 16 & 10 & 3 & $92.50 \%$ \\
Control & 42 & 9 & 6 & 18 & 9 & $78.57 \%$ \\
\hline
\end{tabular}

Notes: After statistical analysis there was significant difference between the two groups of total effective rate. $(P=0.038<0.05)$

\subsection{Comparison of ALT and AST in the serum of patients}

Table 2. Comparison of the serum ALT and AST between the two groups ( $\overline{\mathrm{X}} \pm \mathrm{S} U / \mathrm{L})$.

\begin{tabular}{lllllll}
\hline Liver & \multicolumn{3}{c}{ Test group } & \multicolumn{2}{c}{ Control group } \\
\cline { 2 - 7 } function & $\mathrm{n}$ & Pre-treatment & Post-treatment & $\mathrm{n}$ & Pre-treatment & Post-treatment \\
\hline ALT & 40 & $103.18 \pm 40.04$ & $46.91 \pm 29.17$ & 42 & $97.14 \pm 54.89$ & $52.57 \pm 31.75$ \\
AST & 40 & $82.01 \pm 40.79$ & $43.25 \pm 13.52$ & 42 & $67.79 . \pm 36.81$ & $50.67 \pm 22.89$ \\
\hline
\end{tabular}

Notes: After 12 weeks, the level of ALT and AST in the two groups were significantly improved than before $(P<0.05)$, and the reduction level of AST in the test group was better than the control group $(P<0.05)$. There was no significant difference between the two groups in ALT $(P>0.05)$. 


\subsection{Comparison of degrees of liver function between two groups}

Table 3. Comparison of degrees of the liver function between the two groups.

\begin{tabular}{|c|c|c|c|c|c|c|c|c|c|}
\hline \multirow{2}{*}{ Group } & \multicolumn{3}{|c|}{ Pre-treatment } & \multirow{2}{*}{ Total } & \multicolumn{3}{|c|}{ Post-treatment } & \multirow{2}{*}{ Total } & \multirow{2}{*}{$P$} \\
\hline & $\mathrm{I}$ & II & III & & $\mathrm{I}$ & II & III & & \\
\hline Test & 32 & 8 & 0 & 40 & 37 & 3 & 0 & 40 & \\
\hline Control & 32 & 10 & 0 & 42 & 36 & 6 & 0 & 42 & 0.85 \\
\hline Total & 64 & 18 & 0 & 82 & 73 & 9 & 0 & 82 & \\
\hline
\end{tabular}

Notes: I represents mild damage, II represents moderate damage, III represents severe damage.

After 12 weeks in test group, the number of patients with mild liver function damage increased from 32 to 37 , and the number of patients with moderate damage reduced from 8 to 3 . While in the control group, the number of patients with mild liver function damage increased from 32 to 36, and the number of patients with moderate damage reduced from 10 to 6 . There was no significant difference between the two groups in the liver function improvement rate $(P>0.05)$. In general, it seems to show a tendency that the change of these values in test group is better than that in control group.

\subsection{Comparison of quantitative reduction of $\mathrm{HBeAg}$ and $\mathrm{HBsAg}$ in serum of $\mathrm{CHB}$ patients}

Table 4. Comparison of amount of HBeAg ( $\overline{\mathrm{x}} \pm \mathrm{s}, \mathrm{PEI} U / \mathrm{ml})$ and $\operatorname{HBs} \operatorname{Ag}(\overline{\mathrm{x}} \pm \mathrm{s}, \mathrm{ng} / \mathrm{ml})$ between two groups.

\begin{tabular}{llllllc}
\hline \multirow{2}{*}{ Items } & \multicolumn{3}{c}{ Test group } & \multicolumn{2}{c}{ Control group } \\
\cline { 2 - 7 } & $\mathrm{n}$ & Pre-treatment & Post-treatment & $\mathrm{n}$ & Pre-treatment & Post-treatment \\
\hline HBeAg & 40 & $96.67 \pm 73.15$ & $53.50 \pm 42.96$ & 42 & $93.57 \pm 99.26$ & $67.88 \pm 53.13$ \\
HBsAg & 40 & $307.73 \pm 114.58$ & $257.47 \pm 109.85$ & 42 & $324.44 \pm 125.27$ & $264.33 \pm 157.45$ \\
\hline
\end{tabular}

Notes: After 12 weeks, $\mathrm{HBeAg}$ and $\mathrm{HBsAg}$ in serum of CHB patients were improved partly than before treatment, but there was no significant difference between groups $(P>0.05)$.

\subsection{Comparison of quantitative reduction of HBV-DNA}

Table 5. Comparison of quantity of HBV-DNA between the two groups $(\log 10(\mathrm{IU} / \mathrm{ml}))$.

\begin{tabular}{lllcl}
\hline \multirow{2}{*}{ Items } & \multicolumn{2}{c}{ Test group $(\mathrm{n}=40)$} & \multicolumn{2}{c}{ Control group $(\mathrm{n}=42)$} \\
\cline { 2 - 5 } & Pre-treatment & Post-treatment & Pre-treatment & Post-treatment \\
\hline HBV-DNA & $(2.17 \pm 3.62) \times 10^{7}$ & $(0.92 \pm 1.75) \times 10^{7}$ & $(3.23 \pm 9.67) \times 10^{7}$ & $(1.23 \pm 2.45) \times 10^{7}$ \\
\hline
\end{tabular}

Notes: After 12 weeks, HBV-DNA was all inhibited partly by drugs than before treatment in both groups, but there was no significant difference between groups $(P>0.05)$.

\subsection{Comparison of total efficacy of drugs on CHB}

Table 6. Comparison of total efficacy between the two groups.

\begin{tabular}{lccccc}
\hline Group(n) & Marked effect & Normal effect & No effect & Total effective rate & $P$ \\
\hline Test $(40)$ & 10 & 18 & 12 & $70.00 \%$ & \multirow{2}{*}{0.83} \\
Control(42) & 9 & 18 & 15 & $64.29 \%$ & \\
\hline
\end{tabular}

Notes: The relieve rate of liver function, $\mathrm{HBeAg}, \mathrm{HBsAg}$, and HBV-DNA of the two groups were all improved than before, but there was no significant difference between the two groups $(P>0.05)$. Overall, it seems to show a tendency that the change of these values in test group is better than that in control group.

\section{Discussion}

Oxymatrine is an active ingredient extracted from herb medicine Sophora. According to reports 
oxymatrine plays an important role in protecting liver cells and stimulating the body cells to produce interferon[19]. Oxymatrine also has the effects of anti-inflammatory, anti-hepatic injury, inhibition of HBV replication and immunomodulatory[20-21]. Since 2005, matrine was recommended to treat CHB according to Guideline on chronic hepatitis B prevention and treatment, which was made by Chinese Medical Association Hepatology and Chinese Medical Association infectious diseases[17]. So far, many clinical studies have shown that matrine possesses potential advantages in treat CHB[2225]. In this trail we have choosed matrine as control drug and expected to further confirm and evaluate the efficacy of LCFG for CHB patients and HBV. As shown in Table 1, TCM symtom improvement in test group is better than in control group $(P<0.05)$. The results of this trail showed that there was no significant difference between the LCFG and matrine capsule in trems of reduction of serum $\mathrm{HBeAg}$, HBsAg, HBV-DNA and ALT in CHB patients $(P>0.05)$ (Table2, 3, 4, 5). However, the reduction level of AST in the test group was better than the control group $(P<0.05)($ Table2). At the last, the comparison of total efficacy (the summary of comparision of AST, ALT, HBV-DNA, HbeAg and HBsAg) between the two groups is not significantly (Table6). In a word, it seems to show a tendency that the change of these values in LCFG group is better than that in matrine group.

LCF consists of seven commonly used herbs including Solanum nigrum L., Radix Bupleuri, Hedyotis diffusa Wild, Sedum Sarmentosum, Scutellaria baicalensis, Fructus Gardeniae, Radix Glycyrrhizae. According to the prescription composition of TCM theory, Solanum nigrum L. and Hedyotis diffusa Wild are considered as sovereign medicine, their medicinal property of bitter-cold exerts a certain function of heat-clearing and detoxifying. Sedum Sarmentosum and Fructus Gardeniae are considered as minister medicine, their medicinal property of sweet-cool and bitter-cold can assist minister medicine to exert function removing dampness, detoxifying and clearing heat. Radix Bupleuri and Scutellaria baicalensis are considered as assistant medicine, the combination of two herbs can unobstructe liver-Qi, harmonize stomach-Qi, dry dampness and clear heat, this function is similar to Xiaochaihu Tang recorded in Shanghanlun. Radix Glycyrrhizae was used to be guide medicine in this formula and could reconcile the property of other drugs.

The whole formula possesses the function of heat-clearing, detoxifying, removing dampness and soothing liver-Qi. Recently, many TCM studies suggest that the appearance of CHB is most attributed to infection of dampness-heat and epidemic toxin, coupled with deficiency of healthy Qi. Multiple pathogenic factor could cause dampness-heat mixed with epidemic toxin, and gradually lead to imbalance of viscera, Qi and blood, and dysfunction of body's defenses. Some theories on pathogenesis of CHB in TCM are got more attention[26], including toxin theory, theory of deficiency of vital Qi, theory of stagnancy of damp-heat and theory of multiple reason (toxin, vital Qi deficiency, Qi stagnation, blood stasis tangled with each other). In our opinion[27], dampness-heat and epidemic toxin are the leading factors that cause $\mathrm{CHB}$, liver-stagnation and spleen-deficiency is an important step to CHB. LCF has the effect of heat-clearing and detoxifying, harmonizing spleen and stomach precisely basing on the CHB pathogenesis of toxin invasion, vital Qi deficiency and Qi stagnation in TCM.

So far, it was widely reported that single medicine herb from LCF has the hepatoprotective and antiviral effect at home and abroad. For example, Solanum nigrum extract can protect liver against the $\mathrm{CCl}_{4}$-induced oxidative damage in rats, and has the antiviral potential against $\mathrm{HCV}, \mathrm{HBV}$ and HIV[28-30].Ursolic acid extracted from Hedyotis diffusa Wild possesses hepatoprotective ability and can inhibit HBV replication by blocking the HBx[31].Saikosaponins, the main active constituents of Radix Bupleuri, have been shown to possess immunomodulatory, hepatoprotective, anti-tumor and anti-viral activities, and Saikosaponin c exhibits anti-HBV activity and Saikosaponin d possesses potent cytotoxicity against human hepatocellular carcinoma cells[32]. The extracts of Sedum sarmentosum showed strong hepatoprotective effect on D-galactosamine-induced cytotoxicity in primary cultured mouse hepatocytes[33]. Baicalin extracted from Scutellaria radix showed hepatoprotective effect on acetaminophen-induced liver injury in mice[34]. A kind of glycoprotein isolated from Gardenia jasminoides Ellis can ameliorate liver function[35].

The above papers have reported that the hepatoprotective effect and antiviral activity of single medicine herb from LCF in terms of modern pharmacological mechanism. The microscopic 
mechanisms and therapeutic targets of LCF may be closely relation to the active ingredients included in LCF, and pharmacological mechanism of LCF treating CHB remains will be further studied in the future.

\section{References}

1. Lavanchy D. J Viral Hepat. 11(2): 97-107(2004).

2. Perz JF, Armstrong GL, Farrington LA, Hutin YJ, Bell BP. J Hepatol. 45(4): 529-538(2006).

3. Lai CL, Ratziu V, Yuen MF, Poynard T. The Lancet. 362(9401): 2089-2094(2003).

4. Wu GY, Chen HS. World J Gastroenterol. 13(6): 830-836(2007).

5. Alvarado-Esquivel C, de la Ascensión Carrera-Gracia M, Conde-González CJ, JuárezFigueroa L, Ruiz-Maya L, Aguilar-Benavides S, Torres-Valenzuela A, Sablon E. J Antimicrob Chemother. 57(2):221-223(2006).

6. Lu JJ, Chen EQ, Yang JH, Zhou TY, Liu L, Tang H. Virol J vol. 9: 10-15(2012).

7. Zeng XX, Bian ZX, Wu TX, Fu SF, Ziea E, Woon WT. Am J Chin Med. 39(6): 10611074(2011).

8. Ceylan-Isik AF, Fliethman RM, Wold LE, Ren J. Curr Diabetes Rev. 4(4): 320-328(2008).

9. Wang G, Zhang L, Bonkovsky HL. Chin J Integr Med. 18(4): 253-255(2012).

10. Cui X, Wang Y, Kokudo N, Fang D, Tang W. Biosci Trends. 4(2): 39-47(2010).

11. Lu SH. Clinical Research on Treating chronic hepatitis B of Liver depression and spleen deficiency, heat resistance permits with Longchai decoction [D]. Nanjing University of Chinese Medicine(Chin). (2009).

12. Liu QZ. Journal of Nanjing University of TCM(Chin). 22(1):55-57(2006).

13. Wu XY, Zhu FS. Chinese Journal of Basic Medicine in TCM(Chin). 15(6): 469-470(2009).

14. Xue BY, Wu XY, Yao L, Lu SH, Zhu FS. Journal of Liaoning University of TCM(Chin). 12(12): 10-11(2010).

15. Su KL, Zhu FS. 2012 International Symposium on Information Technology in Medicine and Education [A]. 845-850(2012).

16. Zhu FS. Modem Digestion\&Intervention(Chin). 17(6): 334-337(2012).

17. Chinese Society of Liver Disease and Infectious Diseases, Chinese Medical Association. Guidance for prevention and treatment of chronic hepatitis B. Chin J Hepatol(Chin). 13: 881-891(2005).

18. Zheng XY. Beijing: China Medico-pharmaceutical Sciences and Technology Publishing House(Chin) [A]. 143-151(2002).

19. Li P, Si WK, Wang Y, Yao J. Chinese Journal of Biochemical Pharmaceutics(Chin). 25(5): 261-264(2004).

20. Liu M, Liu XY, Cheng JF. China Journal of Chinese Materia Medica(Chin). 28(9): 801804(2003).

21. Liu N, Kan QC, Zhang XJ, Xv YM, Zhang S, Zhang GX, Zhu L. Exp Mol Pathol. 97(3):470-476(2014).

22. Huang XL. China medicine and pharmacy(Chin). 2(4): 96-97(2012).

23. Gu XB,Yang XJ,Wang D. Pharmacology and Clinics of Chinese Materia Medica(Chin). 26(2): 75-77(2010).

24. Wang ZY, Li GS, Huang HX. Chinese Journal of Integrated Traditional and Western Medicine (Chin). 26(8): 681-684(2006).

25. Zhang XW, Hu GQ, Li CY. World Chinese Journal of Digestology(Chin). 13(3): 317 $320(2005)$.

26. Liang LR, Liang JX. Haerbin Medical Journal(Chin). 24(4): 42(2004).

27. Wang P, Zhu FS. Jiangsu Journal of Traditional Chinese Medicine. 43(11): 76-77(2011).

28. Lin HM, Tseng HC, Wang CJ, Lin JJ, Lo CW, Chou FP. Chemico-Biological Interactions. 
171(3): 283-293(2008).

29. Javed T, Ashfaq UA, Riaz S, Rehman S, Riazuddin S. Virology Journal. 8: 26-32(2011).

30. Halim VA, Muck A, Hartl M, Ibáñez AJ, Giri A, Erfurth F, Baldwin IT, Svatos A. Proteomics. 9(1): 171-181(2009).

31. Wu HY, Chang CI, Lin BW, Yu FL, Lin PY, Hsu JL, Yen CH, Liao MH, Shih WL. Journal of Agricultural and Food Chemistry. 59(5): 1713-1722(2011).

32. Chiang LC, Ng LT, Liu LT, Shieh DE, Lin CC. Planta Medica. 69(8): 705-709(2003).

33. Ninomiya K, Morikawa T, Zhang Y, Nakamura S, Matsuda H, Muraoka O, Yoshikawa M. Chem Pharm Bull(Tokyo). 55(8): 1185-1191(2007).

34. Jang S, Kim HJ, Hwang KM, Jekal SJ, Pae HO, Choi BM, Yun YG, Kwon TO, Chung HT, Kim YC. Immunopharmacol Immunotoxicol. 25(4): 585-594(2003).

35. Lee SJ, Oh PS, Lim KT. Clinical and Experimental Pharmacology and Physiology. 33(10): 925-933(2006). 